\title{
PERFORMANCE ANALYSIS OF MC-CDMA COMMUNICATION SYSTEMS OVER NAKAGAMI-M ENVIRONMENTS
}

Joy long-Zong Chen

Assistant Proffessor, Department of Marketing and Distribution Management, Overseas Chinese Institute of Technology, Taichung, Taiwan, R.O.C., jchen@mail.dyu.edu.tw

Follow this and additional works at: https://jmstt.ntou.edu.tw/journal

Part of the Computer Engineering Commons

\section{Recommended Citation}

Chen, Joy long-Zong (2006) "PERFORMANCE ANALYSIS OF MC-CDMA COMMUNICATION SYSTEMS OVER NAKAGAMI-M ENVIRONMENTS," Journal of Marine Science and Technology. Vol. 14: Iss. 1, Article 6.

DOI: $10.51400 / 2709-6998.2079$

Available at: https://jmstt.ntou.edu.tw/journal/vol14/iss1/6

This Research Article is brought to you for free and open access by Journal of Marine Science and Technology. It has been accepted for inclusion in Journal of Marine Science and Technology by an authorized editor of Journal of Marine Science and Technology. 


\title{
Short Paper
}

\section{PERFORMANCE ANALYSIS OF MC-CDMA COMMUNICATION SYSTEMS OVER NAKAGAMI-M ENVIRONMENTS}

\author{
Joy Iong-Zong Chen
}

Key words: MC-CDMA system, uncorrelated channels, correlated channels, Nakagami-m fading.

\begin{abstract}
The performance for a MC-CDMA (multi-carrier coded-division multiple-access) system working in uncorrelated and correlated fading channels with Nakagami distribution is investigated in this paper. We adopt the BPSK modulation scheme with an alternative expression for Q-function to evaluate the BER (bit error rate) performance of the MC-CDMA system. The sum of Gamma variates is adopted for deriving a closed-form solution for arbitrarily correlated channel in order to avoid the difficulty of explicitly obtaining the pdf (probability density function) for the SNR (signal-to-noise ratio) at the MRC (maximum ratio combining) output. The results with simple expression obviously show that the BER performance of the MC-CDMA system is sensitive to the correlation of fading channels.
\end{abstract}

\section{INTRODUCTION}

Due to the advantages of spectrum efficient, interference immune, high date rate, and insensitivity to frequency selective channel, etc., multiple access system bases on direct sequence CDMA (coded-division multiple-access) has drawn recent interest in the application of wireless radio systems [15]. Especially, multicarrier CDMA (MC-CDMA) appears to be a considerable candidate for future mobile radio communication system.

The BER (bit error rate) analysis of MC-CDMA based on considering different kinds of assumptions, so far, have been dedicated in numerous researches in advance [4, 14, 15]. The performance evaluation of MC-CDMA over multipath fading channels was studied

Paper Submitted 10/07/04, Accepted 05/05/05. Author for Correspondence: Joy Iong-Zong Chen. E-mail: jchen@mail.dyu.edu.tw.

*Department of Communication Engineering, Da Yeh University, No. 112, Rd. Shan-Jeau, Da-Tsuen, Chang-Hwa, Taiwan 51505, R.O.C. in [14]. The results presented in [4] are for uplink channel using MRC (maximal ratio combining) with the assumed frequency offsets condition in correlated fading. The performance of MC-CDMA in non-independent Rayleigh fading was studied in [8]. In [10], where is by use of the method of CF (characteristic function) and residue theorem to calculate the performance of downlink MC-CDMA systems. Both of the envelopes and phases correlation are considered in [11], in which the authors evaluate the performance of a MC-CDMA system operates in Rayleigh fading channel. The literature of [5] assumed that the transmission channel is working in Nakagami-m fading channel, and the postdetection of EGC (equal gain combining) was considered illustrated the error probability of MC-CDMA systems. Recently, the publication shown in [2] evaluated the performance of MC-DS-CDMA system with band interference working in Nakagami fading channels.

In this paper, the generic expression of BER performance for MC-CDMA system working in both uncorrelated and correlated fading channels is evaluated. The general correlation of channels with Nakagami- $m$ fading distribution is assumed. There is a closed-form obtained via the sum of Gamma variates to avoid the difficulty of explicitly obtaining the pdf for the SNR (signal-to-noise ratio) at the MRC output. The results show up that how the factors of channel correlation do affect the performance of MC-CDMA systems.

The rest of this paper is organized as follows: section II gives a description of the MC-CDMA system model. The uncorrelated and correlated Nakagami fading channel models are given in section III. Section IV describes the receiver model of MC-CDMA system. The performance of MC-CDMA operating in uncorrelated and correlated fading cannel is carried out in section V. Numerical results are shown in section VI. Finally, section VII draws conclusions briefly. 


\section{SYSTEM MODEL}

The MC-CDMA system model is described in this section. It is assumed that there exist $K$ simultaneously users with $N$ subcarriers within a single cell. Any effect of correlation among users is going to be ignored by assuming the number of user is uniform distributed. As shown in Figure 1, a signal data symbol is replicated into $N$ parallel copies. The signature sequence chip with a spreading code of length $L$ is used to BPSK (binary phase shift keying) modulated each of the $N$ subscriers of the $k$-th user, where the subcarrier has frequency $C /$ $T_{b} \mathrm{~Hz}$, and where $C$ is an integer number $[14,15]$. The technical described above is like an OFDM (orthogonal frequency division multiplexing) performed on a direct sequence spread-spectrum signal when set $C=1$. The larger values of $C$, the more transmitting bandwidth needed. The transmitted signal, $S_{k}(t)$, of the $k$-th user for the resulting transmitted baseband signal corresponding to the $M$ data bit size can be expressed as

$$
S_{k}(t)=\sqrt{\frac{2 P}{N}} \sum_{m^{\prime}=0}^{M-1 N-1} \sum_{n=0} a_{k}[n] b_{k}\left[m^{\prime}\right] P_{T b}(t) \operatorname{Re}\left[e^{j \omega_{n} t}\right]
$$

where both $a_{k}[n]$ and $b_{k}\left[m^{\prime}\right]$ belong to $\{-1,1\}, P$ is the power of data bit, $M$ denotes the number of data bit, $N$ expresses the number of subcarriers, the sequences $a_{k}$ $[0], \ldots, a_{k}[N-1]$ and $b_{k}[0], \ldots, b_{k}[M-1]$ represent the signature sequence and the data bit of the $k$-th user, respectively. The $P_{T_{b}}(t)$ is defined as an unit amplitude pulse that is non-zero in the interval of $\left[0, T_{b}\right]$, and Re $[\cdot]$ denotes the real part of a complex number, $\omega_{n}=2 \pi\left(f_{c}\right.$ $+n C / T_{b}$ ) is the angular frequency of the $n$-th subcarrier, where $f_{c}$ indicates the carrier frequency, $T_{b}$ is symbol duration.

A frequency-selective channel with $1 / T_{b} \ll B W_{c}$

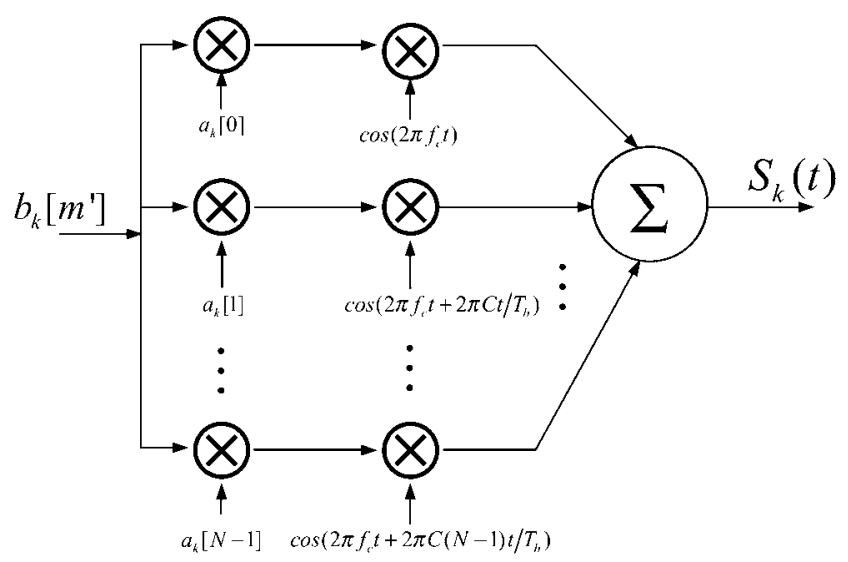

Fig. 1. The transmitter model of the MC-CDMA system.
$<<C / T_{b}$ is addressed in this paper, where $B W_{c}$ is the coherence bandwidth. This channel model means that each modulated subcarrier does not experience significant dispersion and with transmission bandwidth of 1/ $T_{b}$, i.e. $T_{b} \times T_{d}$, where $1 / T_{b}$ is the Doppler shift typically in the range of $0.3 \sim 6.1 \mathrm{~Hz}$ [15] in the indoor environment, and the amplitude and phase remain constant over the symbol duration $T_{b}$. Besides, the channel of interest has the transfer function of the continuous-time fading channel assumed for the $k$-th user can be represented as

$$
H_{k}\left[f_{c}+n \frac{C}{T_{b}}\right]=\beta_{k, n}\left(\cos \theta_{k, n}+j \sin \theta_{k, n}\right)
$$

where $\beta_{k, n}$ and $\theta_{k, n}$ are the random amplitude and phase of the channel of the $k$-th user at frequency $f_{c}+n\left(C / T_{b}\right)$. In order to follow the real world case, the random amplitude, $\beta_{k, n}$ are assumed to be a set of $N$ correlated identically distributed in one of our scenarios.

\section{CHANNEL MODEL}

In this section, there are two cases of propagating channels considered, that is, uncorrelated and correlated Nakagami channels. The equal fading severities are considered for all of the channels, namely $m_{i}=m, i$ $=0, \ldots, N-1$. The pdf of the fading amplitude for the $k$-th user with $n$-th channel, $\beta_{k, n}$, are assumed as r.v. (random variable) with the Nakagami distribution, and given as [7]

$$
P(\beta)=\frac{2 \beta^{2 m-1}}{\Gamma(m)} \cdot\left(\frac{m}{\Omega}\right)^{m} \cdot \exp \left(\frac{m \beta^{2}}{\Omega}\right), \beta \geq 0
$$

where $\Gamma(\cdot)$ is the gamma function defined by $\Gamma(x)=\int_{0}^{\infty} t^{x-1} e^{-t} d t, \Omega=E\left[\beta^{2}\right]$ denotes the average power of the fading signal, the fading figure, $m$, of the amplitude distribution characterizes the severity of the fading, and it is defined as

$$
m=\frac{\Omega^{2}}{E\left[\left(\beta^{2}-\Omega\right)^{2}\right]} \geq 0.5
$$

It is well known that $m=0.5$ (one-sided Gaussian fading) corresponds to worst case fading condition, $m=$ 1 and $m=\infty$ correspond to Rayleigh fading (purely diffusive scattering) and the non-fading conditions, respectively. Next, we consider these two cases.

\section{Uncorrelated channels}

First, if the propagation channels are assumed as $i$. i. $d$ (identically independent distributed), then by use of the variable changing, the variable $\gamma$ is assigned as the 
fading power of the channel. The pdf of $\gamma$ is given follows as a gamma distribution, can be obtained by the processing of random stochastic as

$$
P_{\gamma}(\gamma)=\frac{\gamma^{m-1} e^{-\gamma / \Omega}}{\Gamma(m) \Omega^{m}}
$$

where $\gamma$ is considered as the instantaneous power of the fading amplitude, that is, $\gamma=\beta^{2}$ is assumed.

\section{Correlated channels}

Let $\left[\gamma_{i}\right], i=0, \ldots, N-1$ be a set of $N$ correlated identically distributed, and all the figure parameters and the average power are assumed equivalent, that is, $m_{i}=$ $m_{j}=m$, and $\Omega_{i}=\Omega_{j}=\Omega$, where $i \neq j$, for $i, j=0, \ldots, N-$ 1. The power at the output of the MRC is a function of the sum of the squares of signal strengths, and is given as $R=\sum_{i=0}^{N-1} \gamma_{i}$. Hence following the results extended from Alouini et al. [1] and Moschopoulos [6], the pdf of the output of the MRC receiver, can be expressed as

$$
P_{R}(\gamma)=\prod_{i=0}^{N-1}\left(\frac{\lambda_{1}}{\lambda_{i}}\right) \sum_{v=0}^{\infty} \frac{\mu_{v} \gamma^{m N+v-1} e^{-\gamma / \lambda_{1}}}{\lambda_{1}^{m N+v} \Gamma(m N+v)}
$$

where the coefficients $\mu_{v}$ can be obtained recursively by the following formula given as

$$
\left\{\begin{array}{l}
\mu_{0}=1 \\
\mu_{v+1}=\frac{m}{v+1} \sum_{i=1}^{v+1}\left[\sum_{j=1}^{N}\left(1-\frac{\lambda_{1}}{\lambda_{j}}\right)^{i}\right] \mu_{v+1-i}, v=0,1,2, \ldots
\end{array}\right.
$$

where $\lambda_{1}=\min \left\{\lambda_{i}\right\}$, and $\lambda_{i}, i=0, \ldots, N-1$ are the eigenvalues of the matrix $\underline{Z}=\underline{X Y}$, where $\underline{X}$ is the $N \times N$ diagonal matrix with the entries of average power $\Omega_{i}, i$ $=0, \ldots, N-1$ when the subcarrier paths are correlated, the entries of $\Omega_{i}$ can be obtained by taking the minimum value of $\Omega_{i}=\gamma_{i} / m_{i}$. The matrix $\underline{Y}$ is the $N \times N$ positive definite matrix defined by

$$
\underline{Y}=\left[\begin{array}{cccc}
1 & \rho_{12}^{1 / 2} & \cdots & \rho_{1 N}^{1 / 2} \\
\rho_{21}^{1 / 2} & 1 & \cdots & \rho_{2 N}^{1 / 2} \\
\vdots & \vdots & \ddots & \vdots \\
\rho_{N 1}^{1 / 2} & \cdots & \cdots & 1
\end{array}\right]_{N \times N}
$$

where $\rho_{i j}$ denotes the correlation coefficient between $\gamma_{i}$ and $\gamma_{j}, i \neq j, i, j=0,1,2, \ldots, N-1$, and $\rho_{l m}$ can be expressed as

$$
\rho_{i j}=\frac{\operatorname{Cov}\left(\gamma_{i}, \gamma_{j}\right)}{\left[\operatorname{Var}\left(\gamma_{i}\right) \cdot \operatorname{Var}\left(\gamma_{j}\right)\right]^{1 / 2}}=\rho_{i j}, 0 \leq \rho_{i j} \leq 1
$$

where $\operatorname{Var}(\cdot)$ and $\operatorname{Cov}(\cdot)$ are the variance and the covariance operators, respectively.

\section{MC-CDMA RECEIVER MODEL}

As shown in Figure 2, for $K$ active transmitters, the received signal $r(t)$ can be written as

$$
\begin{aligned}
r(t) & =\sqrt{\frac{2 P}{N}} \sum_{k=0}^{K-1} \sum_{m^{\prime}=0}^{M-1} \sum_{n=0}^{N-1} \beta_{m^{\prime}, n} a_{k}[n] b_{k}\left[m^{\prime}\right] P_{T_{b}}(t \\
& \left.-m^{\prime} T_{b}-\tau_{k}\right) \cos \left(\omega_{n} t+\theta_{m^{\prime}, n}\right)+n(t)
\end{aligned}
$$

where $n(t)$ is the AWGN (additive white Gaussian noise) with a double-sided power spectral density of $N_{0} / 2$. Assuming that acquisition has been accomplished for the user of interesting $(k=0)$. For the reason of using MRC, it is assumed that perfect phase correction can be obtained, i.e., $\hat{\theta}_{0, n}=\theta_{0, n}$. With all the assumptions for MRC combining, the decision variable $N_{0}$ of the $l$-th data bit reference user, is given by

$$
\begin{aligned}
D_{0} & =\frac{1}{T_{b}} \int_{l T_{b}}^{(l+1) T_{b}} r(t) \times \sum_{n=0}^{N-1} a_{0}[n] \times d_{0, n} \\
& \times \operatorname{Re}\left[e^{\left(\omega_{n} t+\theta_{0, n}\right)}\right] d t \\
& =U_{S}+I_{M A I}+\eta_{0}
\end{aligned}
$$

where $r(t)$ is the received signal shown in Eq. (10), $d_{0}$, ${ }_{n}$ is the gain factor for MRC diversity, and $U_{S}$ represents the desired signal, can be expressed as,

$$
U_{S}=\sqrt{\frac{P}{2 N}} \sum_{n=0}^{N-1} \beta_{0, n}^{2} \cdot b_{0}\left[m^{\prime}\right]
$$

and the second term, $I_{M A I}$, is the MAI (multiple access

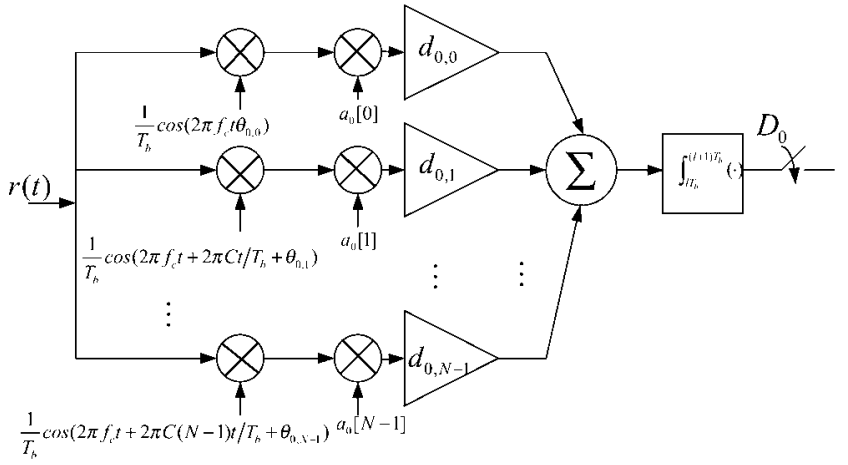

Fig. 2. The receiver model of the MC-CDMA system. 
interference) contributed from all other users which can be written as

$$
\begin{aligned}
I_{M A I} & =\sqrt{\frac{P}{2 N}} \sum_{k=1}^{K-1 N-1} \sum_{n=0} a_{k}[n] \cdot b_{k}\left[m^{\prime}\right] \cdot a_{0}[n] \cdot \beta_{k, n} \\
& \cdot \cos \left(\theta_{k, n}^{\prime}\right)
\end{aligned}
$$

where $\theta_{k, n}^{\prime}=\theta_{0, n}-\theta_{k, n}$ and $\theta_{k, n}$ are $i$. $i$. $d$ uniformly distributed over $[0,2 \pi]$, is the AWGN term.

\section{PERFORMANCE ANALYSIS}

A generalized average BER for the $k$-th user using coherent BPSK (binary phase shift keying) modulation scheme is derived in this section. For coherent demodulation in the presence of AWGN, the probability of error conditioned on the instantaneously SNR can be expressed as [9]

$$
P_{e}(s)=\frac{\Gamma\left(\frac{1}{2}, \sigma_{0} s\right)}{2 \sqrt{\pi}}=0.5 Q\left(\sqrt{2 \sigma_{0} s}\right)
$$

where the incomplete gamma function is defined as $\Gamma(z, t)=\int_{t}^{\infty} x^{(z-1)} e^{-x} d x$, and in order to determine the $S N R$ in last equation, the received instantaneously $S N R$ $\sigma_{0} s$, which conditioned on $\gamma_{0, n}=\beta_{0, n}^{2}$, at output of the receiver is calculated as

$$
\frac{U_{s}^{2}}{\sigma_{T}^{2}}=\frac{\frac{P}{2 N} \sum_{n=0}^{N-1} \beta_{0, n}^{2}}{\sigma_{I_{M A I}^{2}}^{2}+\sigma_{\eta}^{2}}
$$

where $\sigma_{I_{M A I}}^{2}$ is the variance of $I_{M A I}$, which is shown in Eq. (13). In the limiting case of large $N$ and by the methods of central limit theory (CLT), the MAI can be approximated by a Gaussian r.v. with zero mean and the variance, $\sigma_{I_{M A I}}^{2}$, can be determined as [5]

$$
\begin{aligned}
\sigma_{I_{M A I}}^{2} & =E\left[I_{M A I}^{2}\right]=\frac{P}{2}(k-1) \cdot E\left[\beta_{k, n}^{2}\right] \cdot E\left[\cos ^{2} \bar{\theta}_{k, n}\right] \\
& =\frac{P}{4}(k-1) \Omega_{k, n}
\end{aligned}
$$

where $\Omega_{k, n}=E\left[\beta_{k, n}^{2}\right], E\left[\cos ^{2} \bar{\theta}_{k, n}\right]=1 / 2$. On the other hand, the background noise term $\eta_{0}$ is a random variable with zero mean and the variance can be calculated as

$$
\sigma_{\eta_{0}}^{2}=E\left[\eta_{0}^{2}\right]=\frac{N N_{0}}{4 T_{b}}
$$

By substituting Eqs. (16) and (17) into Eq. (15), which can be obtained as

$$
\frac{U_{s}^{2}}{\sigma_{T}^{2}}=\frac{\frac{1}{2 N} \cdot S}{\sigma_{0}}
$$

where $S=\sum_{n=1}^{N-1} \beta_{0, n}^{2} / \Omega_{k, n}$

and $\sigma_{0}=\frac{N N_{0}}{4 P T_{b} \Omega_{k, n}}+\frac{k-1}{4}=\frac{N}{4 \gamma_{0}}+\frac{k-1}{4}$

where $\gamma_{0}=\frac{P T_{b} \Omega_{k, n}}{N_{0}}=\frac{E_{b} \Omega_{k, n}}{N_{0}}$ is the SNR of each bit, and $E_{b}=P T_{b}$ denotes the bit energy.

It is known that the decision variable in Eq. (11) has a Gaussian distribution conditioned on the uncorrelated and correlated channel power $\beta_{0, n}^{2}$, respectively, and the AWGN, $\eta_{0}$, and the MAI, $\eta_{M A I}$ are mutually independent. Therefore, the probability of error by means of BPSK modulation conditioned on the instantaneously SNR has been given in Eq. (15) can be evaluated as follows.

\section{Uncorrelated channels}

We adopt the alternative expression for the $Q$ function, which is expressed as [13]

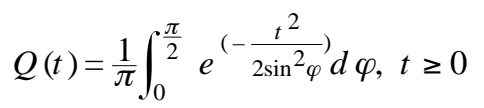

and the random variables $\left\{\gamma_{i}, i=0, \ldots, N-1\right\}$ are assumed to be independent. Such that the Eq. (14) can be expressed as [3]

$$
P_{e}(s)=\frac{1}{\pi} \int_{0}^{\frac{\pi}{2}} \prod_{n=1}^{N} J_{0, n}\left(\Omega_{0, n}, \varphi\right) d \varphi
$$

where the symbol $J_{0, n}\left(\Omega_{0, n}, \varphi\right)$ stands for an integral function, can be determined as

$$
\begin{aligned}
& J_{0, n}\left(\Omega_{0, n}, \varphi\right)=\int_{0}^{\infty} e^{\left(-\frac{\gamma 0, n}{\sin ^{2} \varphi} P_{r}\left(r_{0, n}\right) d r_{0, n}\right.} \\
& =\left(\frac{m}{\Omega_{0, n}}\right)^{m}\left(\frac{1}{\sin ^{2} \varphi}+\frac{m}{\Omega_{0, n}}\right)^{-m}
\end{aligned}
$$

where $\Omega_{0, n}$ indicates the average value of $\gamma_{0, n}$. In Nakagami- $m$ fading channels, the $\gamma_{0, n}$ follows the gamma distribution expressed as in Eq. (5). All $N$ subcarriers are assumed $i . i$. $d$, and the average bit error probability can be calculated by using the simple form of a single integral with finite limits and obtained as

$$
P_{e}=\frac{1}{\pi} \int_{0}^{\frac{\pi}{2}}\left[J_{0, n}\left(\Omega_{0, n}, \varphi\right)\right]^{N} d \varphi
$$

\section{Correlated channels}

The condition of correlated channels are considered in this section, and the average bit error probability for the case can be calculated by averaging Eqs. (6) and 
(14), and yield as

$$
\begin{aligned}
P_{e} & =\int_{0}^{\infty} P_{e \mid s} \cdot P_{e}(S) d S \\
= & \frac{1}{2 \sqrt{\pi}} \prod_{n=0}^{N-1}\left(\frac{\lambda_{1}}{\lambda_{n}}\right)^{m} \sum_{v=0}^{\infty} \frac{\mu_{v}}{\lambda_{1}^{m N+v} \Gamma(m N+v)} \\
& \cdot \frac{\sigma_{0}^{1 / 2} \cdot \Gamma\left(m N+v+\frac{1}{2}\right)}{(m N+v)\left(\sigma_{0}+\frac{1}{\lambda_{1}}\right)^{m N+v+\frac{1}{2}}} \\
& \times{ }_{2} F_{1}\left(1, m N+v+\frac{1}{2} ; m N+v+1 ; \frac{1 / \lambda_{1}}{\sigma_{0}+1 / \lambda_{1}}\right)(25)
\end{aligned}
$$

where $\mu_{v}$ is shown in Eq. (7), $\lambda_{1}$ and $\sigma_{0}$ are shown in Eqs. (6) and (20), respectively, and the symbol ${ }_{2} F_{1}(.$, .; .; .) denotes the confluent hyper-geometric function [9].

\section{NUMERICAL RESULTS}

Some of the numerical results based on Eqs. (22) and (25) are shown in this section. In Figures 3 and 4, the results of SNR (in $\mathrm{dB}$ ) and user number versus BER for MC-CDMA system works over uncorrelated fading channels are illustrated, respectively. On the other hands, Figures 5 and 6 show the BER performance results for MC-CDMA system works in correlated channels environments. In Figure 3 where is not only the different fading parameters, $m=2$ and 4, figure out the different BER performances curves but the different subcarrier numbers, $N=8,16,32$. The results presented that the much more subcarrier the better BER performance. The user number, $K$, versus BER for MCCDMA system operate in uncorrelated fading channels

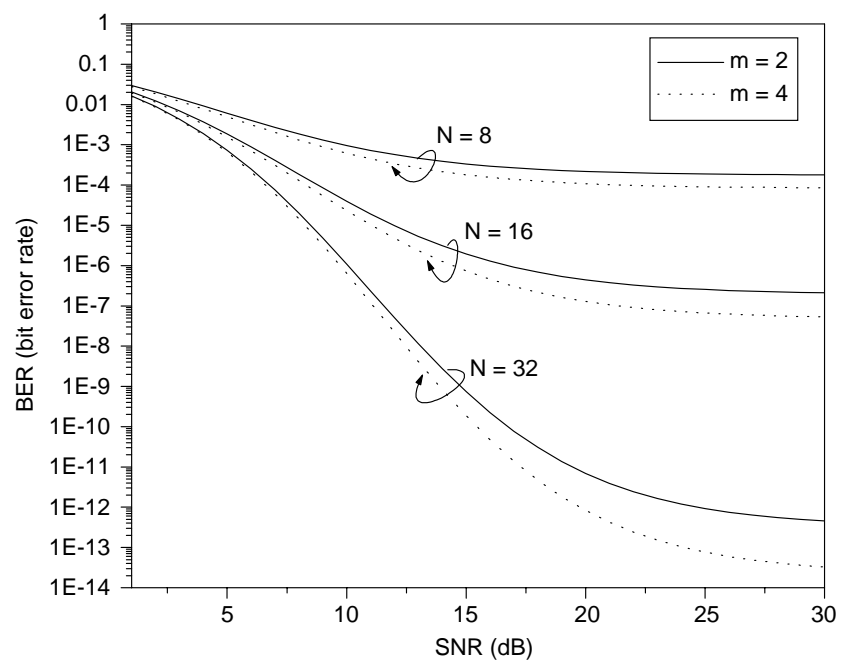

Fig. 3. The SNR vs BER for MC-CDMA system with uncorrelated channels. is illustrated in Figure 4, in which the $S N R=5 d B$ is assumed. The user number is limited both of the SNR and the subcarrier number. The results shown in Figures 3 and 4 can be validated by the previously works as [12].

Next, the different correlation coefficients between the correlated channels are assumed to be $\rho=0.1,0.4$, and 0.8 , which are adopted from [1] (the values of $\rho=$ $0.01,0.16$, and are applied in [1], but the square root is the effective values for putting into the covariance matrix (8)), the subcarrier number and the user number are assumed corresponding to 16 and 8 in Figure 5. To calculate the BER, the mean power of the desired signal is assumed equal to the mean power of each interfering mobile unit. Base on the results shown in Figure 5, we should know that the performance of BER will be pro-

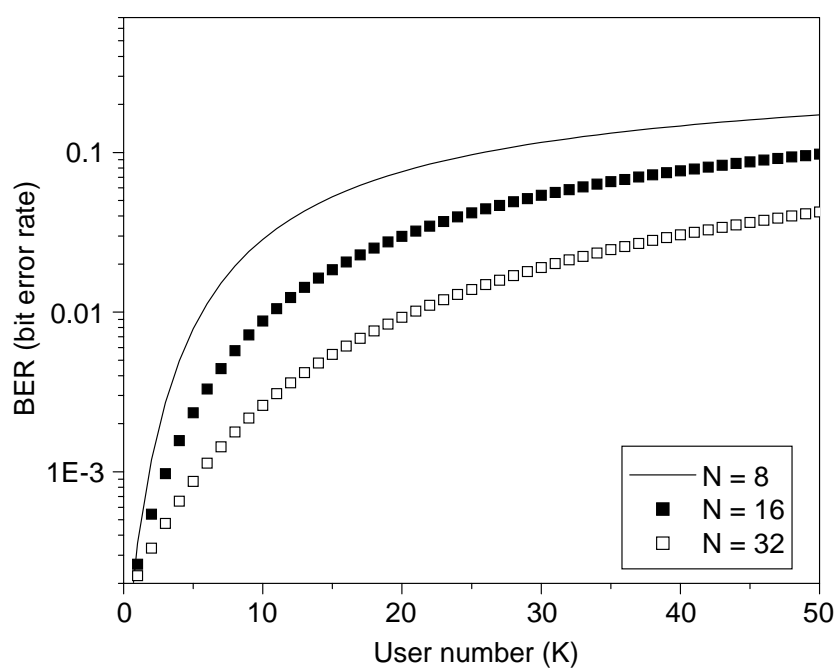

Fig. 4. The user number vs BER for MC-CDMA system with uncorrelated channels.

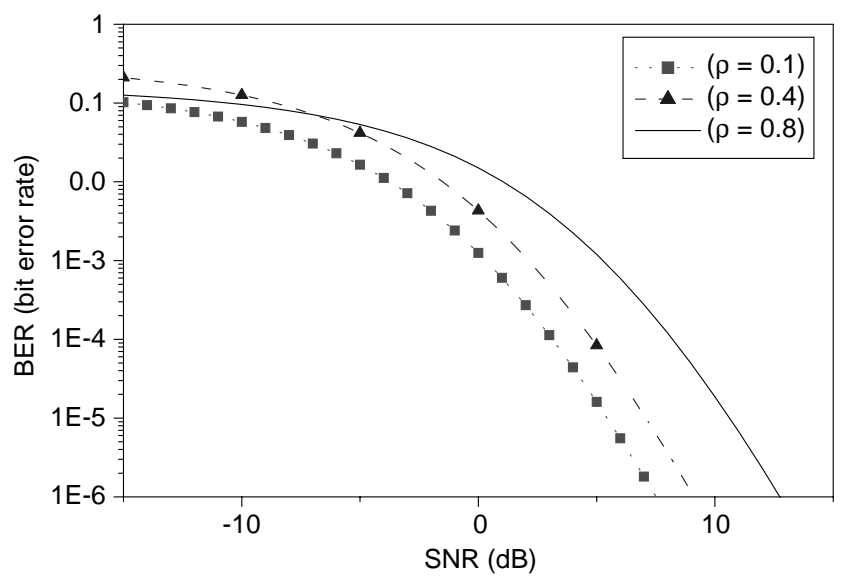

Fig. 5. The SNR vs BER for MC-CDMA system with the correlated fading channels with $N=16, k=8$. 


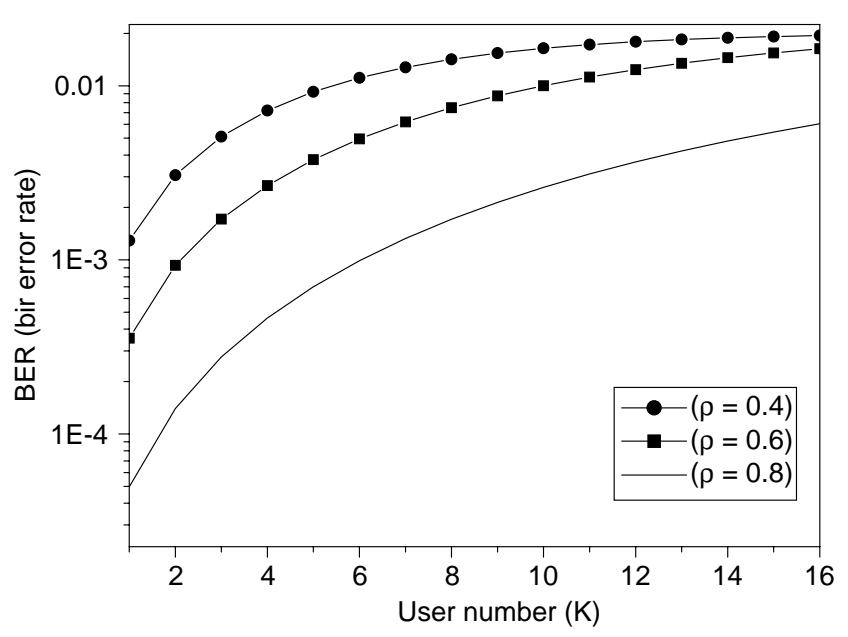

Fig. 6. The user number vs BER for MC-CDMA system with correlated fading channels.

moted gradually by the increasing of the correlation coefficient, $\rho$, between the correlated channels. It is reasonable that the system performance BER becomes much better when the fading parameter is increasing. The performance of BER versus user number is shown in Figure 6, in which the correlation coefficients are assumed as, $\rho=0.4$ and 0.8 , the average bit SNR is set as $5 \mathrm{~dB}$ [11]. From the figure it should be noted that the impact of channel correlation definitely affects the performance of MC-CDMA system.

\section{CONCLUSION}

The SNR and the user number versus probability error rate for MC-CDMA works in uncorrelated and correlated Nakagami-m fading channels has been evaluated in this paper. Some new equations were derived in this research, for example, the expression of the BER was calculated by the sum of Gamma variates for the shake of avoiding the difficulty ways. The results explicitly show that the phenomena of channel correlation do degrade the performance of MC-CDMA communication systems. Therefore the consideration of correlation coefficient for channel fading should pay much attention while designing the MC-CDMA systems for wireless radio systems.

\section{REFERENCES}

1. Alouini, M.-S., Abdi, A., and Kaveth, M., "Sum of Gamma Variates and Performance of Wireless Communication Systems over Nakagami-Fading Channels," IEEE Transactions on Vehicular Technology, Vol. 50, No. 6, pp. 1471-1480 (2001).
2. Chen, J.I.Z., "Performance Evaluation of MC-DS-CDMA in Nakagami Fading Channels Including Partial Band Interference," Journal of Science and Technology, Vol. 14, No. 1, pp. 27-37 (2005).

3. Grodshteyn, I.S. and Ryzhik, I.M., Table of Integrals, Series, and Products, Academic Press, San Diego, CA (1994).

4. Kim, T., Kim, Y., Park, J., Ko, K., Choi, S., Kang, C., and Hong, D., "Performance of an MC-CDMA System with Frequency Offsets in Correlated Fading," Proceedings of IEEE International Conference on ICC 2000, Vol. 2, pp. 1095-1099 (2000).

5. Li, Z. and Latva-Aho, M., "Error Probability for MCCDMA in Nakagami-m Fading Channels Using Equal Gain Combining," Proceedings of IEEE International Conference on ICC 2002, Vol. 1, pp. 227-231 (2002).

6. Moschopoulos, P.G., "The Distribution of the Sum of Independent Gamma Random Variables," Annals of the Institute of Statistical Mathematics- Part A, Vol. 37, pp. 541-544 (1985).

7. Nakagami, M., "The m-Distribution-A General Formula of Intensity Distribution of Rapid Fading," In Hoffman, W.C. (Eds.), Statistic Methods of in Radio Wave Propagation, Pergamon Press, New York, pp. 3-36 (1960).

8. Park, J., Kim, J., Choi, S., Cho, N., and Hong, D., "Performance of MC-CDMA Systems in Non-independent Rayleigh Fading," Proceedings of IEEE International Conference on Communications, Vol. 1, pp. 506510 (1999).

9. Schwartz, M., Bennett, W.R., and Stein, S., Communication Systems and Techniques, McGraw-Hill, New York (1966).

10. Shi, Q. and Latva-Aho, M., "Exact Error Floor for Downlink MC-CDMA with Maximal Ratio Combining in Correlated Nakagami Fading Channels," Proceedings of 2002 International Zurich Seminar on Broadband Communications, pp. 37-1-37-5 (2002).

11. Shi, Q. and Latva-Aho, M., "Performance Analysis of MC-CDMA in Rayleigh Fading Channels with Correlated Envelopes and Phases," IEE ProceedingsCommunications, Vol. 150, No. 3, pp. 214-220 (2003).

12. Shiro, K. and Milstein, L.B., "Performance of Multicarrier DS CDMA Systems," IEEE Transactions on Communications, Vol. 44, No. 2, pp. 238-246 (1996).

13. Simon, M.K. and Alouini, M.-S., "A Unified Approach to the Performance Analysis of Digital Communication over Generalized Fading Channel," Proceeding of the IEEE, Vol. 86, No. 9, pp. 1860-1877 (1998).

14. Sourour, E.A. and Nakagawa, M., "Performance of Orthogonal Multicarrier CDMA in a Multipath Fading Channel," IEEE Transactions on Communications, Vol. 44, pp. 356-367 (1996).

15. Yee, N., Linnartz, J.-P., and Fettweis, G., "Multi-carrier CDMA in Indoor Wireless Radio Networks," IEICE Transactions on Communications, Vol. E77-B, No. 7, pp. 900-904 (1994). 